\title{
3-Dimensional Chemical Structures of an Isolated Cell Nucleus by a Scanning Transmission X-ray Microscope
}

Takuji Ohigashi $^{1,2, *}$, Atsushi Ito $^{3}$, Kunio Shinohara ${ }^{3}$, Shigenobu Tone ${ }^{4}$, Yuichi Inagaki ${ }^{1}$, Hayato Yuzawa $^{1}$ and Nobuhiro Kosugi ${ }^{1,2}$

1. UVSOR Synchrotron, Institute for Molecular Science, Okazaki, Japan

2. The Graduate University for Advanced Studies, Okazaki, Japan.

3. School of Engineering, Tokai University, Hiratsuka, Japan.

4. Tokyo Denki University, Hatoyama, Japan

*Corresponding author, ohigashi@ims.ac.jp

Apoptosis is a programmed cell death which is frequently observed in many biological processes such as developmental process. Nuclear condensation and DNA fragmentation occur during execution of apoptosis, and this process is classified into 3 stages. Apoptosis is generally observed by using fluorescence microscope with staining process [1] but high resolution image, chemical compositions and 3-dimensional (3D) structure are still unclear. A scanning transmission X-ray microscopy (STXM) is a promising technique to elucidate these issues. STXM can obtain 2-dimensional distribution of chemical states with high spatial resolution around $30 \mathrm{~nm}$ by using near edge X-ray absorption fine structure (NEXAFS). Our group has been investigating process of apoptosis and a biological cell by using STXM [2-4]. In these years, we have been developing computed tomography (CT) to perform 3D spectroscopy by changing the energy of the X-ray [5]. In this study, as a preliminary measurement, 3D distributions of protein and DNA of a cell nucleus at normal stage (i.e. stage 0 ) were observed.

As the sample, isolated nuclei from HeLa S3 cells were attached on a slide glass using cell adhesive agent, Cell-Tak (Fisher Scientific), and were fixed by glutaraldehyde. After critical point drying process, the isolated cell nucleus was glued on a tip of a tungsten needle by using a crystal bond under an optical microscope with micro-manipulators. The sample was mounted on a sample cell with a rotational axis $[5]$.

Experiment was performed at STXM beamline, BL4U, in UVSOR Synchrotron (Okazaki, Japan). BL4U is equipped with an in-vacuum soft X-ray undulator and a monochromator with a varied line spacing plane grating. By using this combination, the energy range of the $\mathrm{X}$-rays from 75 to $800 \mathrm{eV}$ is currently available with energy resolution $(\mathrm{E} / \Delta \mathrm{E})$ of $\sim 5,000$. STXM uses a Fresnel zone plate (FZP) as a focusing device and spatial resolution down to $30 \mathrm{~nm}$ is available. To perform CT by using this system, a focal depth of the FZP should be longer than thickness of the sample to assume paths through the sample as a parallel beam. Therefore, the FZP with smaller NA was used instead of higher spatial resolution. Details of the FZP are as follows; diameter of $240 \mu \mathrm{m}$, outermost zone width of $45 \mathrm{~nm}$, Ta pattern on a $\mathrm{Si}_{3} \mathrm{~N}_{4}$ membrane. The focal depth of this FZP is $5.2 \mu \mathrm{m} @ 400 \mathrm{eV}$ and is nearly enough length for a cell nucleus. 
In this study, we focused on observing 3D distribution of DNA and protein in the cell nucleus. NEXAFS spectra of DNA and histone as protein around N K-edge are shown in Fig.1. These spectra show remarkable difference on $1 \mathrm{~s}-\pi^{*}$ transition so that they were used as reference spectra. In CT measurement, 41 X-ray transmission images (energy stack) were acquired by changing the $\mathrm{X}$-ray energy from 397 to $410 \mathrm{eV}$. 50 energy stacks were acquired by rotating the sample $3.6^{\circ}$ each $\left(180^{\circ}\right.$ rotation in total). Then, a size of one image was $8 \times 8$ $\mu \mathrm{m}^{2}$ with $50 \times 50$ pixels and a dwell time was $2 \mathrm{~ms}$.

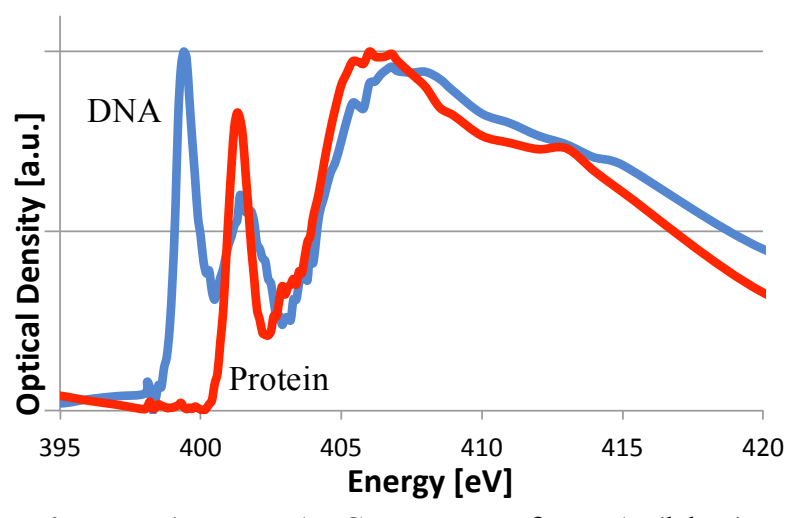

Figure. 1. NEXAFS spectra of DNA (blue) and protein (red) at $\mathrm{K}$ absorption edge of nitrogen

For reconstruction of 3D image, the X-ray transmission images of the energy stack were aligned and 2D distributions of DNA and protein were obtained by fitting their reference spectra to each energy stack by using aXis2000 software [6]. Sinograms were extracted from data sets of the 2D distributions of DNA and protein respectively and cross sectional images were reconstructed by home-made filtered backprojection algorithm. The reconstructed 3D distribution of DNA and 2D distribution of DNA and protein are shown in Fig. 2. While the 2D distribution of DNA in Fig. 2(b) shows nucleoli (shown by arrows) and compartment like structures in the nucleus, that of protein in Fig. 2(c) shows only the compartment like structures and nearly no protein in the nucleoli. However, the reconstructed image in Fig. 2(c) is low contrast and has circle artifact around the image. These degradations are caused by error in fitting of the reference spectra of protein. Therefore, we have been discussing contribution of other molecules to the cell nucleus [4]. This method will be applied to the cell nuclei in the other stages of apoptosis.
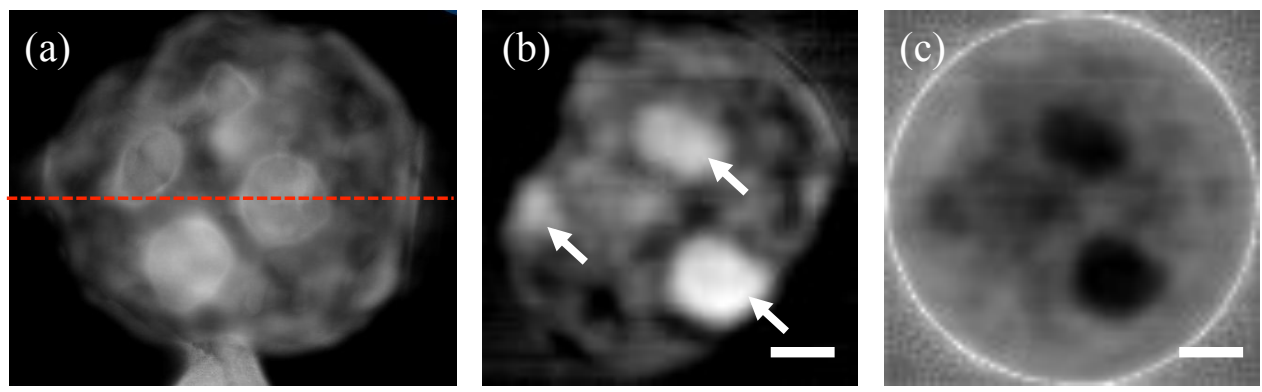

Figure. 2. Distributions of DNA and protein of the isolated nucleus of a HeLa S3 cell. A 3D volume projection image of DNA (a) and a cross sectional images of DNA (b) and protein (c) (position is shown in (a) by a dotted line), respectively. Bright color shows high density. Scale bars are $2 \mu \mathrm{m}$.

\section{References:}

[1] S. Tone et al, Exp. Cell Res. 313 (2007) 3635-3644.

[2] T. Ohigashi et al, AIP Conf. Proc. 1696 (2016) 020027.

[3] K. Shinohara, et al, J. Phys.: Conf. Ser. 849 (2017) 012003.

[4] K. Shinohara et al, submitted.

[5] T. Ohigashi et al, J. Phys.: Conf. Ser. 849 (2017) 012044.

[6] http://unicorn.mcmaster.ca/aXis2000.html 